\title{
Modality-Specific Working Memory Systems Verified by Clinical Working Memory Tests
}

\author{
Eun-Hee Park ${ }^{1,2}$, Duk-In Jon ${ }^{1}$ \\ ${ }^{1}$ Department of Psychiatry, Hallym University College of Medicine, Anyang, ${ }^{2}$ Department of Psychology, Seoul National University, Seoul, Korea
}

\begin{abstract}
Objective: This study was to identify whether working memory (WM) can be clearly subdivided according to auditory and visual modality. To do this, we administered the most recent and universal clinical WM measures in a mixed psychiatric sample.

Methods: A total of 115 patients were diagnosed on the basis of DSM-IV diagnostic criteria and with MINI-Plus 5.0, a structured diagnostic interview. WM subtests of Korean version of Wechsler Adult Intelligence Scale-IV and Korean version of Wechsler Memory Scale-IV were administered to assess WM. Confirmatory factor analysis (CFA) was used to observe whether WM measures fit better to a one-factor or two-factor model.

Results: CFA results demonstrated that a two factor model fits the data better than one-factor model as expected. Conclusion: Our study supports a modality model of WM, or the existence of modality-specific WM systems, and thus poses a clinical significance of assessing both auditory and visual WM tests.
\end{abstract}

KEY WORDS: Modality-specific working memory; WAIS-IV; WMS-IV.

\section{INTRODUCTION}

Working memory (WM) is a cognitive system that allows for transient storage and manipulation of given information. ${ }^{1)}$ From a clinical perspective, WM is fairly important. Deficits in WM are reported in many psychiatric diseases as WM is deeply related to clinical symptoms, other complex cognitive functions, and activities of daily living. ${ }^{2-5)}$

WM model proposed by Baddeley ${ }^{6-8)}$ is one of the most influential WM models and consists of two domain-specific systems and a domain-general system. Domain-specific systems were divided into phonological loop and visual-spatial sketchpad according to modalities of information and thus is often called as modality-specific systems. Each subsystem has separate and independent capacity-

Received: July 4, 2017 / Revised: September 6, 2017

Accepted: September 10, 2017

Address for correspondence: Duk-In Jon, MD, PhD

Department of Psychiatry, Hallym University Sacred Heart Hospital, Hallym University College of Medicine, 22 Gwanpyeong-ro

170beon-gil, Dongan-gu, Anyang 14068, Korea

Tel: +82-31-380-3752, Fax: +82-31-381-3751

E-mail: cogni@hallym.or.kr

ORCID: https://orcid.org/0000-0002-1565-7940 limited storage for temporal retention of verbal or visual-spatial information. However, the subsystems are not perfectly independent and are controlled by the central executive, a domain-general system.

In general, WM tests require the subjects to manipulate the given auditory or visual information all the while maintaining them. Clinicians frequently use psychometric tools such as WM subtests of Wechsler Intelligence or Memory Scales to measure WM capacity. Furthermore, recent versions of Wechsler Scales ${ }^{9,10)}$ have placed greater emphasis on WM components than their predecessors. Auditory and visual WM tests were included separately in Wechsler Adult Intelligence Scale-IV (WAIS-IV) ${ }^{9)}$ and Wechsler Memory Scale-IV (WMS-IV) ${ }^{10)}$. Routinely measuring both forms of WM is not recommended since they are strongly inter-correlated and functionally similar. ${ }^{11)}$ Regardless, investigation is required to identify whether it is sufficient to only administer WM measures of a single modality. Therefore, our study is intended to investigate which of one or two factor model (OFM; TFM) fits better with WM test data from the newest versions of Wechsler Scales in a heterogeneous psychiatric sample. We predicted that WM has modality distinctive constructs (i.g.,

(c) This is an Open-Access article distributed under the terms of the Creative Commons Attribution Non-Commercial License (http://creativecommons.org/licenses/by-nc/4.0) which permits unrestricted non-commercial use, distribution, and reproduction in any medium, provided the original work is properly cited. 
possible TFM).

\section{METHODS}

\section{Participants}

Participants were psychiatric out- and in-patients aged 19 to 60 years who visited Hallym University Sacred Heart Hospital from November 2015 through October 2016. Patients were diagnosed by psychiatrists based on the Diagnostic and Statistical Manual of Mental Disorders 4th edition (DSM-IV) ${ }^{12)}$ diagnostic criteria. A structured diagnostic interview, MINI International Neuropsychiatric Interview Plus 5.0 (MINI-Plus 5.0), ${ }^{13,14)}$ and psychological tests were conducted by clinical psychology trainees under the supervision of a licensed clinical psychologist. Patients with physical disabilities or other severe medical conditions were excluded from the study, ultimately resulting in a total of 115 participants. Patients' demographic characteristics are presented in Table 1. Written patient consent and research approval by Hallym University Sacred Heat Hospital institutional review board were obtained (IRB No. 2015-I097).

\section{Measures}

WM subtests of Korean version of WAIS-IV (K-WAIS$(\mathrm{V})^{15)}$ were administered to assess auditory WM: digit span and letter number sequencing (LNS). Each sub-scores of digit span test (forward, backward, and sequential) was coded separately for statistical analysis. WM subtests of Korean version of WMS-IV (K-WMS-IV) ${ }^{16)}$ were administered to assess visual WM. Spatial addition is required to maintain and manipulate spatial information, while symbol span is required to maintain and manipulate visual details.

\section{Statistical Analysis}

Expectation-maximization algorithm ${ }^{17)}$ was conducted to replace missing data. The estimates of parameters were obtained using maximum likelihood methods. Confirmatory factor analysis (CFA) tested whether psychometric tasks developed to measure WM fit better to OFM or TFM in a mixed clinical sample. Various indices were used to evaluate model fit. ${ }^{18,19)}$ The $\chi^{2}$ test was utilized to assess the fit of hypothetical models to the sample data. If $\chi^{2}$ value is small and its significance is more than 0.10 , it indicates good fit. However, since $\chi^{2}$ statistics is sensitive to sample size, additional indices were to be considered. Our study used Comparative Fit Index (CFI), Root Mean Square Error of Approximation (RMSEA), Tucker-Lewis Index (TLI), and Standardized Root Mean Square Residual (SRMR) as alterative indices. Hu and Bentler ${ }^{18)}$ suggested that RMSEA $<0.06$, CFI $>0.95$, TLI $>0.95$, and SRMR $<0.08$ indicate good fit for continuous data. IBM SPSS version 20.0 and IBM SPSS AMOS version 22.0 (IBM Co., Armonk, NY, USA) were used for data analysis.

\section{RESULTS}

OFM tested the fit of an overall WM factor that encompassed all obtained WM tests. If OFM demonstrated good fit, all measurements may represent the same or unitary construct of WM. Correlated TFM tested whether two WM factors can be separated by modality yet related to each other. The results are presented in Figure 1. In our sample, TFM showed good fit in all fit indices whereas OFM showed good fit in only a few. Furthermore, TFM revealed a significantly better fit to our data than did OFM; $\chi^{2}$-difference $(1)=6.23, p<0.05$. In short, our result shows that two modalities of WM tests are related to each

Table 1. Demographic information

\begin{tabular}{|c|c|c|c|}
\hline Charcateristic & Gender (F\%) & Education (yr) & Age (yr) \\
\hline Schizophrenia $(n=18)$ & 72.222 & $12.278 \pm 2.653$ & $30.444 \pm 11.868$ \\
\hline Major depressive disorder $(\mathrm{n}=39)$ & 33.333 & $13.000 \pm 1.821$ & $27.667 \pm 11.577$ \\
\hline Bipolar disorder $(n=6)$ & 33.333 & $13.000 \pm 5.292$ & $28.333 \pm 13.604$ \\
\hline Anxiety disorder $(n=24)$ & 33.333 & $14.210 \pm 2.377$ & $32.667 \pm 14.095$ \\
\hline Developmental disorder ( $n=13)$ (mental retardation, autism) & 38.462 & $10.408 \pm 3.250$ & $28.231 \pm 12.050$ \\
\hline Neurocognitive disorder $(n=10)$ & 20.000 & $11.400 \pm 4.881$ & $43.800 \pm 16.457$ \\
\hline Others $(n=5)$ & 20.000 & $13.400 \pm 1.950$ & $23.400 \pm 4.615$ \\
\hline Total $(n=115)$ & 33.913 & $12.870 \pm 3.002$ & $30.461 \pm 13.1751$ \\
\hline
\end{tabular}

Values are presented as mean \pm standard deviation. 


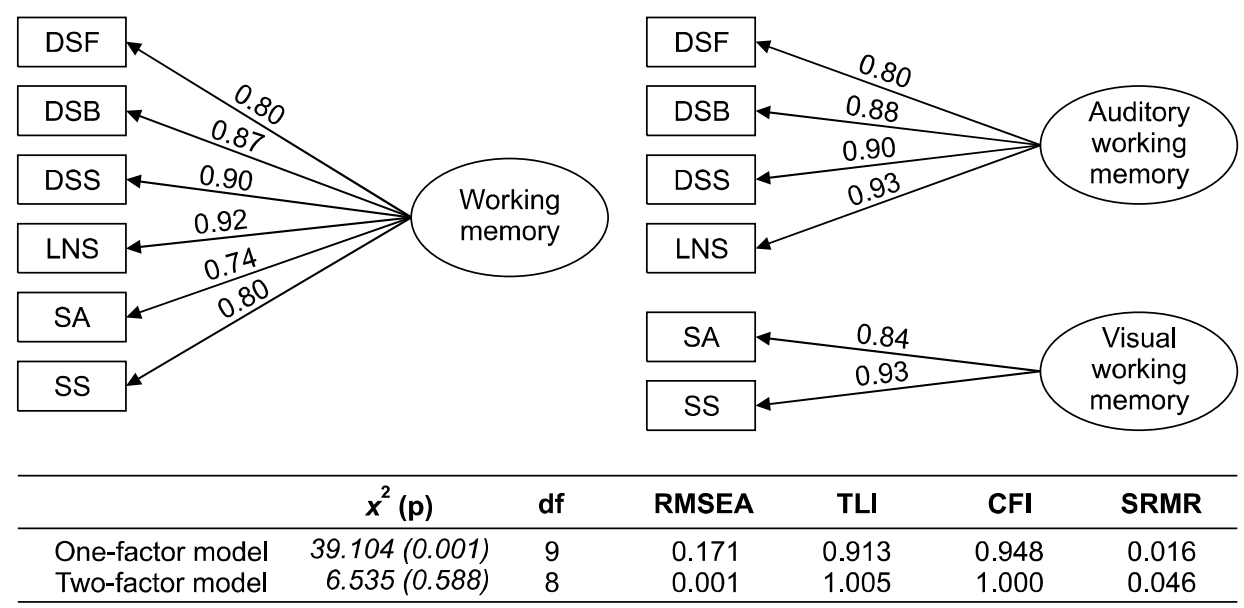

Fig. 1. Confirmatory factor analysis of Korean version of Wechsler Adult Intelligence Scale-IV and Korean version of Wechsler Memory Scale-IV working memory subtests. In the figure, the circles represent latent constructs, and the squares represent the observed variables. Single-headed arrows represent standardized factor loadings and double-headed arrows represent correlations between factors. All parameter estimates are standardized and significant at $p<0.001$.

DSF, digit span forward; DSB, digit span backward; DSS, digit span sequencing; LNS, letter number sequencing; SA, spatial addition; SS, symbol span; df, degree of freedom; RMSEA, Root Mean Square Error of Approximation; TLI, Tucker-Lewis; CFI, Comparative Fit Index; SRMR, Standardized Root Mean Square Residual.

other $(r=0.84, p<0.001)$ but measure independent constructs.

\section{DISCUSSION}

This study was conducted to examine whether WM can be subdivided by modality using the most current and universal clinical WM measures in a mixed sample of psychiatric patients. CFA results demonstrated that TFM fits the data better than OFM as we had expected. This suggests that there are separate and independent WM stores for auditory and visuospatial information, and supports modality-specific WM systems, ultimately posing clinical significance of assessing both auditory and visual WM tests.

Our study also supports a modality model of WM. The results are in line with previous research that showed WM is divisible by modality. ${ }^{20-23)}$ Many clinicians have mainly utilized auditory WM tests rather than visual WM tests unless special cases required them to do without the auditory WM tests. ${ }^{11)}$ However, given our results, clinicians should be cautious when deriving generalized conclusions of WM from a single modality of WM; administering both WM modalities may be more desirable and informative in the clinical setting. Particularly, doing so is important for not only neurological diseases with unilat- eral lesions but also language disorders, non-verbal learning disorders, and attention deficit hyperactivity disorder with domain-specific impairments. ${ }^{23,24)}$ Although a previous study that used CFA on WAIS-IV and WMS-IV, supported both OFM and TFM, ${ }^{25)}$ the study had used a normal standardized sample and included arithmetic but not LNS as a variable reflecting WM construct. These differences may have contributed to the discordant results.

To test the models in CFA, we replaced arithmetic, a core WM subtest, with LNS, a supplemental WM subtest. LNS is considered as the best predictor of WM among other clinical WM measures ${ }^{20,25)}$ and, with respect to the most recently published Wechsler Intelligence Scale for Children, fifth edition (WISC-V), ${ }^{26)}$ LNS along with digit span is a core subtest to make up auditory WM index and arithmetic comprises quantitative reasoning index. On the other hand, existing studies have found arithmetic subtest requires diverse cognitive processes such as attention/concentration, verbal comprehension, and mathematical skills as well as WM. ${ }^{25,27)}$ It also has the lowest factor loading among clinical WM constructs. ${ }^{28)}$

Additionally, our findings revealed how well each WM measures represent its corresponding construct of WM. LNS was the best measure of its WM construct in both OFM and TFM. This endorses the assertion by Shelton et $a^{28)}$ that LNS is the best clinical WM test and has process- 
ing demands most comparable to those of laboratory WM tests. Digit span sequencing (DSS) has factor loading higher than digit span forward (DSF) but similar to digit span backward (DSB). It is consistent with the finding that DSB and DSS similarly require more WM capacity than DSF. ${ }^{11)}$ However, Egeland ${ }^{20)}$ argued that the difference in processing levels among these three sub-tasks are not sufficient to distinguish between passive storage and active executive processing. If this holds true, these sub-tasks might be limited to detect task-specific performance patterns in clinical groups. Further study is required to investigate whether this reflects insufficient differences in WM load, or the tasks being different from what they were originally intended to measure, or the characteristics of the clinical sample. Although visual WM subtests of WMS-IV well represent the construct of visual WM, symbol span seems slightly better to reflect the construct and have slightly higher WM load than spatial addition. However, replication by additional empirical research is necessary.

This study also has some limitations. The study found only content dimension (i.g., modality), not functional dimensions (i.g., storage and processing) of clinical WM tests suggested by Baddeley's model. Also, since the study data is based on various diagnostic groups, the interpretation and generalization of our results may be limited; cross-validation in other types of clinical groups or healthy controls is needed.

In summary, present study has an important clinical implication in that, by demonstrating modality-specific properties of clinical WM tests in a mixed clinical group, it serves as a wake-up call for clinicians who make a hasty decision for deficits in WM based on only single modality of WM measures.

\section{REFERENCES}

1. Baddeley AD. Is working memory still working? Am Psychol 2001;56:851-864.

2. Collette F, Van der Linden M, Salmon E. Executive dysfunction in Alzheimer's disease. Cortex 1999;35:57-72.

3. Fleming K, Goldberg TE, Gold JM, Weinberger DR. Verbal working memory dysfunction in schizophrenia: use of a Brown-Peterson paradigm. Psychiatry Res 1995;56:155-161.

4. Goldman-Rakic PS. Working memory dysfunction in schizophrenia. J Neuropsychiatry Clin Neurosci 1994;6:348357.

5. Pasini A, Paloscia C, Alessandrelli R, Porfirio MC, Curatolo P. Attention and executive functions profile in drug naive $A D H D$ subtypes. Brain Dev 2007;29:400-408.

6. Baddeley AD, Hitch G. Working memory. Psychol Learn Motiv 1974;8:47-89.

7. Baddeley A. Oxford psychology series, No. 11. Working memory. New York:Clarendon Press/Oxford University Press; 1986.

8. Baddeley A. The episodic buffer: a new component of working memory? Trends Cogn Sci 2000;4:417-423.

9. Wechsler D. Wechsler adult intelligence scale-fourth edition (WAIS-IV). San Antonio, TX:Pearson;2008.

10. Wechsler D. Wechsler memory scale-fourth edition (WMS-IV). San Antonio, TX:Pearson;2009.

11. Weiss LG, Saklofske DH, Coalson D, Raiford SE. WAIS-IV clinical use and interpretation: Scientist-practitioner perspectives. London:Academic Press,2010.

12. Association Psychiatric Association. Diagnostic and statistical manual of mental disorders: fourth edition (DSM-IV). Washington, D.C.:American Psychiatric Association; 1994.

13. Sheehan DV, Lecrubier $\mathrm{Y}$, Sheehan $\mathrm{KH}$, Amorim $\mathrm{P}$, Janavs J, Weiller E, et al. The Mini-International Neuropsychiatric Interview (M.I.N.I): The development and validation of a structured diagnostic psychiatric interview for DSM-IV and ICD-10. J Clin Psychiatry 1998;59 Supp/ 20:22-33.

14. Yoo SW, Kim YS, Noh JS, Oh KS, Kim CH, NamKoong K, et al. Validity of Korean version of the Mini-International Neuropsychiatric Interview. Anxiety Mood 2006;2:50-55.

15. Hwang ST, Kim JH, Park KB, Chey JY, Hong SH. Korean-Wechsler adult intelligence scale IV (K-WAIS-IV). Daegu:Korea Psychology Co.;2012.

16. Chey JY, Kim JH, Park KB, Hwang ST, Hong SH. Korean-Wechsler memory scale-IV (K-WMS-IV). Daegu: Korea Psychology Co.; 2011.

17. Dempster AP, Laird NM, Rubin DB. Maximum likelihood from incomplete data via the EMalgorithm. I R Stat Soc Series B Stat Methodol 1977;39:1-38.

18. Hu L, Bentler PM. Cutoff criteria for fit indexes in covariance structure analysis: Conventional criteria versus new alternatives. Struct Equ Modeling 1999;6:1-55.

19. Kline RB. Principles and practice of structural equation modeling. 4th ed. New York:Guilford Press;2015.

20. Egeland J. Measuring working memory with digit span and the letter-number sequencing subtests from the WAIS-IV: too low manipulation load and risk for underestimating modality effects. App/ Neuropsychol Adult 2015;22:445-451.

21. Fougnie D, Marois R. Working memory capacity is modality-specific: Evidence of separate stores for auditory and visuospatial stimuli. J Vis 2008;8:1169.

22. Kessels RP, van den Berg E, Ruis C, Brands AM. The backward span of the Corsi Block-Tapping Task and its association with the WAIS-III Digit Span. Assessment 2008;15:426-434.

23. Martinussen R, Hayden J, Hogg-Johnson S, Tannock R. $A$ meta-analysis of working memory impairments in children with attention-deficit/hyperactivity disorder. J Am Acad Child 
Adolesc Psychiatry 2005;44:377-384.

24. Gooch D, Snowling M, Hulme C. Time perception, phonological skills and executive function in children with dyslexia and/or ADHD symptoms. J Child Psychol Psychiatry 2011; 52:195-203.

25. Holdnack JA, Zhou X, Larrabee GJ, Millis SR, Salthouse TA. Confirmatory factor analysis of the WAIS-IVMMS-IV. Assessment 2011;18:178-191.

26. Wechsler D. Wechsler intelligence scale for children-Fifth ed- ition (MSC-V): Technical and interpretive manual. Bloomington, MN:Pearson Clinical Assessment;2014.

27. Stearns CL, Dunham M, Mclntosh D, Dean RS. Attention deficit/hyperactivity disorder and working memory in clinically referred adults. Int I Neurosci 2004; 114:273-287.

28. Shelton JT, Elliott EM, Hill BD, Calamia MR, Gouvier WD. $A$ comparison of laboratory and clinical working memory tests and their prediction of fluid intelligence. Intelligence 2009; 37:283-293. 